\title{
The Grandview Medical Center Bioethics Consultation Service Perspective on the Peril of Isolated and Vulnerable Individuals due to COVID-19
}

\author{
Nicholas Salupo $^{1}$ (D) $\cdot$ Leland Cancilla $^{1} \cdot$ Sharon Merryman ${ }^{1} \cdot$ Jeffrey Kaufhold ${ }^{1}$ \\ Received: 25 February 2021 / Revised: 11 May 2021 / Accepted: 14 May 2021 / \\ Published online: 20 July 2021 \\ (c) National University of Singapore and Springer Nature Singapore Pte Ltd. 2021
}

\begin{abstract}
We present the perspective of a Bioethics Consultation Service operating in an urban hospital in Dayton, Ohio, USA, as it adapted to treating Sars-CoV-2 patients throughout 2020. Since the first case of COVID-19 was reported in Ohio on 9 March 2020, until 1 January 2021, the Bioethics Consultation Service was consulted 60 times, a $22.5 \%$ increase from the same period of 2019. The most common diagnoses requiring consultation included end-stage renal disease requiring dialysis, out-of-hospital cardiac arrest, and sepsis. Only $10 \%$ of consultations were for patients hospitalized with COVID-19. This is a qualitative analysis of the cases we saw and a discussion of factors that affected our service while adapting to COVID-19 standards of care.
\end{abstract}

Keywords Sars-CoV-2 · COVID-19 · Ethical dilemma · Bioethics consultation · Adult alone

At the beginning of the Sars-CoV-2 (COVID-19) pandemic in the United States of America, we saw a fragmented and disorganized response by hospital systems due to an absence of a unified response from local, state, and federal governments. Subsequently, COVID-19 cases exceeded 27 million with more than 485,000 deaths from COVID-19 in the U.S. by 16 February 2021 (Centers for Disease Control and Prevention 2021). This overwhelming demand on the U.S. healthcare system has identified the weakest parts of the system and forced us, increasingly, to adopt crisis standards of care, leading to an unknown amount of risk and adverse outcomes. From these crisis standards, we have seen Bioethics Consultation Services begin to

Nicholas Salupo

Nicholas.Salupo@ketteringhealth.org

1 Bioethics Consultation Service, Kettering Grandview Medical Center, Dayton, OH, USA 
confront unique questions that have not had to be asked in the twenty-first century. It is the intention of the authors of this paper to present the unique perspective of a Bioethics Consultation Service operating in an urban hospital in a mid-size town (population 140,640) once COVID-19 restrictions were implemented.

Grandview Medical Center is an approximately 350-bed hospital in the Kettering Health Network that serves the western Dayton, Ohio community. Our hospital population is predominantly low income and black, indigenous, people of color (BIPOC), both of which are groups that have seen higher rates of hospitalization and death associated with COVID-19 (NCIRD 2020; National Center for Health Statistics 2021). At the time of writing, our hospital has created a makeshift COVID-19 intensive care unit with a 24-bed capacity, and converted a critical care step-down unit to a COVID-19 general medical floor with a 35-bed capacity. Nurse staffing has been pooled from our medical intensive care unit, surgical intensive care units, and advanced care step-down unit. Physician staffing for these units consists of the hospitalist and critical care services. In addition to the attending physicians, nurse practitioners and internal medicine residents are responsible for responding to rapid response and code blue calls on the COVID-19 units. The Bioethics Consultation Service consists of a physician chairperson certified as a Healthcare Ethics Consultant along with other attending physicians in internal medicine and neurology plus internal medicine residents as well as supporting nursing and administrative staff. Our Bioethics Service will accept and train any physicians willing to participate in Ethics consultations. While we have all struggled with scarce resources, it is the opinion of the authors that the hospital network has done as successful of a job as possible providing the safest possible environment for employees to care for COVID-19 patients.

Since the first case of COVID-19 was reported in Ohio on 9 March 2020, and the first death due to COVID-19 was reported in Ohio on 19 March 2020, until 1 January 2021, our Bioethics Consultation Service was consulted 60 times, a 22.5\% increase from the same period of time in 2019 (Welsh-Huggins and Gillispie 2020). While this data is currently being actively collected and reviewed, this does not appear to be congruent with the expected growth of our service. In addition, our overall hospital volume has been down due to newly implemented distancing and isolation protocols. This would suggest that, with fewer admissions and an objectively larger volume of consults, the COVID-19 pandemic has played a role in increasing the utilization of the Bioethics Consultation Service.

We were surprised to find that only $10 \%$ of the consultations to the Bioethics Consultation Service were for patients hospitalized with a primary diagnosis of COVID-19 or for medium-long range complications of COVID-19. The vast majority of cases were admitted to the medical or surgical intensive care units. The three most common diagnoses included end-stage renal disease requiring dialysis, out-ofhospital cardiac arrest, and sepsis. With COVID-19 restrictions in place, we have seen an increase in patients that are either not established with or unable to schedule an appointment with their primary care physician. We believe this led to delaying necessary care of chronic medical conditions that require constant monitoring, ultimately ending with the patient urgently or emergently requiring hospitalization. Patients at higher risk of death due to Sars-CoV-2 are also hesitant to utilize the 
healthcare system in an attempt to avoid exposure to others that are capable of transmitting COVID-19. Due to the complex and multifactorial relationships of access to healthcare, socioeconomic status, employment food security, education, housing, environment, and social support, BIPOC patients suffered worse renal and cardiac outcomes prior to the COVID-19 pandemic (Longino and Kramer 2020). Along with chronic medical conditions, a higher likelihood of living in crowded housing conditions, being an essential worker incapable of remote work, or relying on a job without sick leave has created a disparity in who receives hospital-based and ambulatory primary care. Paradoxically, in an attempt to protect these individuals, we may actually be further impeding access to medical resources and lowering the level of care they receive compared to their predominantly white, affluent, and privately insured counterparts.

The most common reasons for the involvement of the Bioethics Consultation Service in patients not infected with COVID-19 were to determine an appropriate spokesperson and to aid with the withdrawal of life-sustaining measures. Anecdotally, we do not feel that we have more patients being admitted with life-threatening illnesses or without appointed spokespeople. Rather the isolation of these individuals and the severing of the patient from the family unit while admitted is resulting in an increased utilization of the Bioethics Consultation Service to act as an intermediary or, in some cases, as the surrogate decision maker. For most of the year 2020, our hospital protocol has only allowed for one to two family members to be physically present in the hospital at any given time. As the amount of COVID-19 cases in our hospital network increased, we transitioned to a system that allowed for the same single person to visit a patient for the duration of the hospitalization. By December, we were only allowing family to be present during end-of-life moments. All of these measures, taken to protect both the public and the hospital staff, prevented the care team from having the large family conferences that are typical of patients requiring a Bioethics Service consultation. In several instances, we had family meetings in the hospital parking lot to prevent a large group from being indoors together and unable to observe six-foot distancing practices. Not only have we been unable to convene a group of stakeholders in the patient's care we are now relying, almost exclusively via telephone, on a sole decision maker. Often this has required to follow up, again via telephone, with the sole decision maker because they require time to consult with more family, friends, and spiritual leaders involved in the patient's life. While these restrictions are our best attempt to limit the spread of illness, they certainly act as barriers to communication with families and surrogate decision makers.

We have had relatively few consultations on patients diagnosed with COVID-19, and the cases we have consulted on are largely due to a provision in Ohio law. Ohio state law requires a 48-hour waiting period after the decision is made to remove life-sustaining treatment until hospital staff are able to take any action. COVID-19 consultations consisted almost exclusively of family requests to waive this waiting period. These decisions were approved in all cases based on criteria including pressure ulcers of the face from being placed in a prone position in spite of proper prophylaxis, increasing oxygen requirements on full ventilator support, elevated lactic acid levels suggesting tissue ischemia, and/or other evidence of continued pain or suffering with proper sedation protocols. One consult was placed due to a patient 
requesting intubation in spite of his healthcare proxy refusing invasive ventilation. The dilemma arose when the healthcare proxy demanded the patient, who was capable of making his own medical decisions, be made comfort-care only instead of pursuing appropriately indicated therapy that the patient had requested. Ultimately, the patient required transfer to a tertiary care center for extracorporeal membrane oxygenation, and several hours were spent dispelling COVID-19-related conspiracy theories that the health care proxy held to be true. In several cases, patients and their families refuted the existence of Sars-CoV-2 even after patients would require intubation and mechanical ventilation. Far from novel examples, these specific incidents highlight the political nature of the implementation of public health measures. Politicization has historically affected disease outbreaks from the 1655 bubonic plague in London, to AIDS in the 1980s, and hepatitis C in the 2010s (Newman 2012; Piot et al. 2007; Saab et al. 2018). Unfortunately, during periods of rapid disease transmission (i.e., epidemics or pandemics), there are xenophobic and bigoted responses by large populations that only further complicate the treatment of patients suffering from these deadly diseases. Our Bioethics Consultation Service was not immune to the politically partisan response to COVID-19 or the suffering in extremis caused by COVID-19. It is a primary goal of the authors to maintain a Bioethics Consultation Service that pursues the principles of beneficence, non-maleficence, autonomy, and justice for all patients regardless of race, gender, ethnicity, sexual orientation, and/or political affiliation.

We have been forced to accelerate our evaluation of patients with "missed DNR" orders. In patients presenting to the hospital with COVID-19 requiring intubation, respiratory failure occurs so rapidly that there is limited time to discuss treatment options with both the patient and the patient's appointed decision maker. In several cases, a patient has been intubated or resuscitated, and once the family is contacted, we find the patient had a pre-existing DNR order. These errors have most commonly occurred when a patient comes from home or an extended care facility that does not have ready access to the patient's advance directive. These cases require the Ethics Consultant to weigh the directives of conflicting Ohio laws. One such law requires a 48-hour waiting period prior to withdrawal of life-sustaining treatments, and the other, the Ohio Patient Self Determination Act (DNR) law, allows the patient to preemptively describe their preferences for future health care needs. This has led to several internal quality improvement projects aimed at early identification of advance directives upon presentation and admission to the hospital.

We believe the absence of a spokesperson in cases involving patient's lacking capacity to make their own medical decisions is most likely due to the hospital's visitor ban. Prior to COVID-19, family or appointed health care proxies were able to visit the patient without solicitation from the hospital staff. The importance of accurate copies of advance directives and healthcare power of attorney forms in the electronic medical record provided by outpatient health care providers was acutely noted. Without proper documentation, we could not easily, if at all, contact the individuals capable of honoring the patient's goals of care. We also experienced a growing percentage of older and medically frail patients living alone despite often requiring intensive monitoring and therapy as outpatients. These individuals are mostly dependent on social safety net 
programs that suffered from lack of resources and staffing due to COVID-19. This is consistent with the trend of a growing American populace older than 65 years requiring the majority of U.S. health care resources (Dieleman et al. 2020). This has left us contemplating what specific sociocultural trends may be resulting in fewer adult children not being involved in the care of their sick and disabled parents. Could this be related to the cultural value of independence historically embraced by U.S. Baby Boomers? Perhaps it was related to insufficient family leave resources for those earning the majority of an income for a family. The exact cause is likely complex and multifactorial, but this should not stop us from attempting to address the root cause. We are also looking at possible programs that could be developed to increase the prevalence of adults with advance directives. There has been some attention paid to merely increasing the awareness of the need to have conversations regarding end-of-life decisions, such as that which occurred after the Terry Schiavo case in 2006. However, this is a complex and multifactorial problem that would likely require large shifts in cultural norms. In our patient population, there is also the problematic combination of low levels of formal schooling completed and low levels of medical literacy. While not exclusively due to the COVID-19 pandemic, they have exacerbated the limitations of a health care system that is exclusionary and unequal based on characteristics including ethnicity, sex, gender, income, and education. The authors believe that high-quality health care is a human right and we continue our work to bridge these disparities.

The world has become intimately familiar with guidelines and COVID-19 standards of care changing daily, and it would be naïve to believe that we have a complete understanding of how the Bioethics Community did, and should have, responded to this pandemic and how it will impact our health care systems into the future. This, however, has not stopped us from evaluating the ethical dilemmas we have witnessed and actively had to participate in. Previous reports detail common fundamental ethical questions that were involved in caring for patients during the COVID19 pandemic and made recommendations on ethical actions to be taken (Kramer et al. 2020; Rosenbaum 2020). These recommendations offer a standard of care to be pursued but implementation can often vary from hospital to hospital due to local availability of resources and cultural norms. Our account is unique in that we offer a retrospective categorization of the ethical dilemmas experienced during a global pandemic at a medium-sized community-based academic medical center. After evaluation of our Bioethics Service's consultation data, there appeared to be fundamental ethical trends that occurred in both patients diagnosed with COVID-19 and patients not diagnosed with COVID-19.

First, and anecdotally the most frightening, was creating an allocation process for our scarce resources. Fair distribution of scarce resources under contingency or crisis capacity has always been an integral function of bioethics services (Persad et al. 2009). Our allocation model triaged patients based on age, risk of dying from co-morbid conditions, lifestyle and compliance issues, expected outcome, and the Sepsis-related Organ Failure Assessment (SOFA) score to maximize the benefits produced by scarce resources, treat people equally, promote and reward instrumental value, and give priority to the worst off (Vincent et al. 1996; Emanuel et al. 2020). The triage team should consist of several senior physicians and nurses, a medical ethicist, and specialists as needed. On admission, a patient would be assigned a SOFA 
score calculated from their clinical data at presentation. If the score were greater than 11 , the patient would be managed medically or provided palliative care as this patient would be so critically ill as to be not likely to survive their current illness. SOFA scores less than or equal to 7 were highest priority patients indicating good likelihood of survival. SOFA scores of 8-11 were intermediate priority patients, and if no significant organ failure was present on admission, a patient would be deferred treatment. Patient's SOFA scores were evaluated at 24- and 72-hour intervals to assess for improvement in their condition, and if none was made, then the patient was no longer eligible for resource-intensive care. If a patient were to worsen at this re-evaluation interval, they could either become eligible or disqualified for critical care. This process was able to objectively sort patients into groups that offered the highest chance of survival to those with the best prognosis. It was also easily taught to resident physicians, nurses, and other hospital staff involved in providing critical care. We are fortunate that we ultimately did not have to initiate care rationing due to collaboration among regional hospital facilities with the capacity to absorb the overflow of patients, but the entire network of hospitals did come dangerously close to running out of inpatient beds the week of Christmas 2020. The COVID-19 pandemic has made us realize that all health care facilities should have a plan to provide care should resources become acutely limited and Bioethics Services are uniquely positioned to aid in the development of these policies to provide equal access for all individuals and increase efficiency to maximize outcomes for those receiving care.

The rapid expansion of evidence supporting practices such as mask wearing, distancing of 6 feet $(2 \mathrm{~m})$, and limiting contact with vulnerable individuals has led to health care facilities across the world instituting measures to limit the spread of COVID-19 in the interest of public health (Brooks and Butler 2021; Gandhi et al. 2020; Johansson et al. 2021; Jones et al. 2020; Gopalakrishna et al. 2004). These behavioral modifications plus Sars-CoV-2 vaccines will likely prove to be the path to ending the pandemic spread of COVID-19. However, we have seen a large-scale resistance of these behaviors and medical interventions based on political ideology, mistrust of the healthcare systems, and a variety of falsely held conspiracy theories. As Kopar et al. addressed in their analysis of this problem, patient autonomy is a fundamental ethical principle, but during a pandemic, we must limit obliging certain preferences in the interest of vulnerable and critically ill patients (Kopar et al. 2021). Options for treating patients refusing COVID-19 testing include honoring their request and treating them as presumed negative, physically forcing them to take a COVID-19 test, honoring their request, and treating them as presumed positive, or explain the risk they pose to hospital staff and vulnerable patients and advising them to seek care elsewhere. As a protection for our hospital staff, we developed a protocol that did refuse the right to treatment to anyone that denied to be tested for SarsCoV-2 at admission. To the knowledge of the Bioethics Committee, we did not have any patient who chose to not be admitted to the hospital; however, there was at least one patient that was discharged home instead of to a skilled nursing facility because she denied a repeat Sars-CoV-2 test at the time of discharge. It is ethically compelling to refuse care to an individual that is unwilling to undergo appropriate screening in order to protect health care workers and vulnerable populations. 
For most of the year 2020, our hospital had some form of restriction on visitors. At the height of the pandemic, our hospital protocol completely refused any visitation in the hospital except for end-of-life situations. There have been myriad stories on the psychological and emotional distress this has caused both hospital staff and family members (Siddiqi 2020; Piscitello 2020). These restrictions were intended to protect hospital staff and vulnerable patients hospitalized for illnesses other than COVID-19. As previously described, this caused significant difficulty for the Bioethics Service in protecting patient autonomy and assessing the stakeholders involved in a patient's care. However, we have begun to see reports that these restrictions may not have significantly limited the spread of COVID-19 (Weiner et al. 2020). The bioethics literature would benefit from a thorough retrospective review of these restriction protocols for the development of future best practices. The lack of uniformity and transparency of implementation could perpetuate ethical dilemmas and act in direct contradiction to the intended outcome.

The most common reasons for consultation of our Bioethics Service were for the withdrawal of life-sustaining treatment and for appointing a spokesperson for patients without the capacity to make their own medical decisions. We feel that these two dilemmas are very closely related due to patients being placed on invasive life-sustaining treatments merely because they lack proper understanding and thorough counseling on end-of-life decision making. Accurate expectations may be more commonplace with the removal of the false portrayal of cardiopulmonary resuscitation and life-sustaining treatments in television shows and movies. With increased social awareness of advance directives, we are hopeful that patients will regain autonomy by making their goals of care known prior to urgently or emergently requiring the provision of critical care. Although we have not experienced this trend, there does appear to be some anecdotal data that suggest there has been an increase in advance directives being completed by the general public (Auriemma et al. 2020). Unfortunately, in our patient population, social safety-net resources do not include advance care planning, and it often is overlooked in patients with complex and chronic medical conditions that are also subjected to poverty and systemic racism. We would recommend that all primary care services include end of life or goals of care discussion for older adults or those with chronic medical conditions. All physicians, advanced practice providers, and emergency service providers should routinely be checking code status in all hospitalized patients. Isolated and vulnerable individuals are left to care for themselves and when requiring invasive and intensive medical care they lack the family and community support to appropriately communicate their goals of care with hospital staff. The care of hospitalized patients has been the traditional purview of Bioethics Services, but this may offer an opportunity to expand our services into the ambulatory setting to make patients aware of their rights, and responsibilities, to maintain their integrity. We have identified this as an ongoing priority at our medical center and will be devoting significant time and resources to empower patients to participate as active members of their care team.

In conclusion, this past year has forced us to consider social safety net programs as they apply to medical care. The U.S. healthcare model needs revision, and the longer we delay the innovation, the longer our most vulnerable patients will continue 
to face the brunt of morbidity and mortality outcomes. We have been impacted by the disinformation campaign waged by conspiracy theorists and political extremists. It is our assertion that COVID-19 has accelerated the destabilization of the current healthcare model and failure to address the underlying inequalities causing unnecessary suffering would violate moral and ethical standards of care. At our medical center, we are hurriedly adapting to these changing needs and putting in place protocols that we hope will make positive gains over the coming decade in reducing the health care gaps and increasing social equity both among and between groups in our community. It is our goal that by sharing this with the bioethics community at large, we may work collaboratively to build a more just and compassionate future.

Author Contribution All authors contributed to the conception and design of this work, revised it for intellectual content, and approved the final manuscript.

\section{Declarations}

Ethics Approval This retrospective chart review study involving human participants was completed in accordance with the ethical standards of the institution and national research committee along with the 1964 Helsinki Declaration and its later amendments or comparable ethical standards. The Kettering Health Network Institutional Review Board (IRB) approved this study.

Competing Interests The authors declare no competing interests.

\section{References}

Auriemma, Catherine L., Scott D. Halpern, Jeremy M. Asch, Matthew Van Der Tuyn, and David A. Asch. 2020. Completion of advance directives and documented care preferences during the coronavirus disease 2019 (COVID-19) pandemic. JAMA Network Open 3 (7): e2015762. https://doi.org/ 10.1001/jamanetworkopen.2020.15762.

Brooks, J.T., and J.C. Butler. 2021. Effectiveness of mask wearing to control community spread of SARSCoV-2. JAMA 325 (10): 998. https://doi.org/10.1001/jama.2021.1505.

Centers for Disease Control and Prevention. 2021. "CDC COVID Data Tracker." covid.cdc.gov/ covid-data-tracker/\#cases_casesper100k.

Dieleman, J.L., J. Cao, A. Chapin, C. Chen, Z. Li, A. Liu, et al. 2020. US health care spending by payer and health condition, 1996-2016. JAMA 323 (9): 863-884. https://doi.org/10.1001/jama.2020.0734.

Emanuel, E.J., G. Persad, R. Upshur, B. Thome, M. Parker, A. Glickman, et al. 2020. Fair allocation of scarce medical resources in the time of Covid-19. New England Journal of Medicine 382 (21): 2049-2055. https://doi.org/10.1056/nejmsb2005114.

Gandhi, M., C. Beyrer, and E. Goosby. 2020. Masks do more than protect others during COVID-19: reducing the inoculum of SARS-CoV-2 to protect the wearer. Journal of General Internal Medicine 35 (10): 3063-3066. https://doi.org/10.1007/s11606-020-06067-8.

Gopalakrishna, G., P. Choo, Y.S. Leo, B.K. Tay, Y.T. Lim, A.S. Khan, et al. 2004. SARS transmission and hospital containment. Emerging Infectious Diseases 10 (3): 395-400. https://doi.org/10.3201/ eid1003.030650.

Johansson, M.A., T.M. Quandelacy, S. Kada, P.V. Prasad, M. Steele, J.T. Brooks, et al. 2021. SARSCoV-2 transmission from people without COVID-19 Symptoms. JAMA Netw Open 4(1):e2035057. Published 4 Jan 2021. https://doi.org/10.1001/jamanetworkopen.2020.35057 
Jones, Nicholas R., Zeshan U. Qureshi, Robert J. Temple, Jessica P.J. Larwood, Trisha Greenhalgh, and Lydia Bourouiba. 2020. Two metres or one: what is the evidence for physical distancing in covid19? BMJ 370: m3223. https://doi.org/10.1136/bmj.m3223.

Kopar, P.K., J.B. Kramer, D.E. Brown, and G.V. Bochicchio 2021. Critical ethics: how to balance patient autonomy with fairness when patients refuse coronavirus disease 2019 testing. Critical Care Explorations 3(1). https://doi.org/10.1097/cce.0000000000000326

Kramer, J.B., D.E. Brown, and P.K. Kopar. 2020. Ethics in the time of coronavirus: recommendations in the COVID-19 pandemic. Journal of the American College of Surgeons 230 (6): 1114-1118. https:// doi.org/10.1016/j.jamcollsurg.2020.04.004.

Longino, K., and H. Kramer. 2020. Racial and ethnic disparities, kidney disease, and covid-19: a call to action. Kidney Medicine 2 (5): 509-510. https://doi.org/10.1016/j.xkme.2020.07.001.

National Center for Immunization and Respiratory Diseases (NCIRD) 2020. Division of Viral Diseases. "Coronavirus Disease 2019 (COVID-19)-Associated Hospitalization Surveillance Network (COVID-NET)." Centers for Disease Control and Prevention, Centers for Disease Control and Prevention, www.cdc.gov/coronavirus/2019-ncov/covid-data/covid-net/purpose-methods.html. Accessed 17 Mar 2021.

National Center for Health Statistics. 2021. "Deaths involving coronavirus disease 2019 (COVID-19) by race and Hispanic origin group and age, by state." data.cdc.gov/NCHS/ Deaths-involving-coronavirus-disease-2019-COVID-19/ks3g-spdg.

Newman, K.L. 2012. Shutt up: bubonic plague and quarantine in early modern England. Journal of Social History 45 (3): 809-834. https://doi.org/10.1093/jsh/shr114.

Persad, G., A. Wertheimer, and E.J. Emanuel. 2009. Principles for allocation of scarce medical interventions. Lancet 373 (9661): 423-431. https://doi.org/10.1016/s0140-6736(09)60137-9.

Piot, P., S. Russell, and H. Larson. 2007. Good politics, bad politics: the experience of AIDS. American Journal of Public Health 97 (11): 1934-1936. https://doi.org/10.2105/AJPH.2007.121418.

Piscitello, G. 2020. Opinion: Hospitals should reevaluate restrictive visitation policies during the pandemic. CNN. https://www.cnn.com/2020/12/12/opinions/covid-hospital-visitation-policies-pisci tello/index.html. Accessed 21 Mar 2021.

Rosenbaum, L. 2020. Facing Covid-19 in Italy - ethics, logistics, and therapeutics on the epidemic's front line. New England Journal of Medicine 382 (20): 1873-1875. https://doi.org/10.1056/nejmp 2005492.

Saab, S., L. Le, S. Saggi, V. Sundaram, and M.J. Tong. 2018. Toward the elimination of hepatitis C in the United States. Hepatology 67 (6): 2449-2459. https://doi.org/10.1002/hep.29685.

Siddiqi, H. 2020. To suffer alone: hospital visitation policies during COVID-19. Journal of Hospital Medicine 15 (11): 694-695. https://doi.org/10.12788/jhm.3494.

Vincent, J.-L., R. Moreno, J. Takala, S. Willatts, A. De Mendonça, H. Bruining, et al. 1996. The SOFA (Sepsis-related Organ Failure Assessment) score to describe organ dysfunction/failure. Intensive Care Medicine 22 (7): 707-710. https://doi.org/10.1007/bf01709751.

Weiner, H.S., J.I. Firn, N.D. Hogikyan, R. Jagsi, N. Laventhal, A. Marks, et al. 2020. Hospital visitation policies during the SARS-CoV-2 pandemic. American Journal of Infection Control. https://doi.org/ 10.1016/j.ajic.2020.09.007.

Welsh-Huggins, A., and M. Gillispie. 2020. Ohio announces 3 positive tests as state's $1^{\text {st }}$ virus cases. https://apnews.com/article/e3d23821458b12ba0c461289ba9a7fc1.

Publisher's Note Springer Nature remains neutral with regard to jurisdictional claims in published maps and institutional affiliations. 